\title{
Limiting the explanatory scope of extended active inference: the implications of a causal pattern analysis of selective niche construction, developmental niche construction, and organism-niche coordination dynamics
}

\author{
Regina E. Fabry ${ }^{1}$
}

Received: 3 October 2020 / Accepted: 1 February 2021 / Published online: 15 February 2021

(c) The Author(s) 2021

\begin{abstract}
Research in evolutionary biology and philosophy of biology and cognition strongly suggests that human organisms modify their environment through active processes of niche construction. Recently, proponents of the free-energy principle and variational active inference have argued that their approach can deepen our understanding of the reciprocal causal relationship between organisms and their niche on various scales. This paper examines the feasibility and scope of variational formalisations and conceptualisations of the organism-niche nexus with a particular focus on the extended active inference account. I will draw a conceptual distinction between selective niche construction, developmental niche construction, and organism-niche coordination dynamics and argue that these notions capture different causal patterns, each of which with a distinct scope. Against this background, I will analyse and discuss the extended active inference account and its strategy to integrate variational active inference with work on extended cognition. The proponents of extended active inference assume that their account can provide an explanation of selective niche construction, developmental niche construction, and organism-niche coordination dynamics. However, my key claim will be that this account has the potential to elucidate the workings of organism-niche coordination dynamics, but does not adequately capture the causal patterns of selective niche construction and developmental niche construction.
\end{abstract}

Keywords Free-energy principle $\cdot$ Variational active inference $\cdot$ Selective niche construction · Developmental niche construction · Organism-niche coordination dynamics $\cdot$ Extended cognition

Regina E. Fabry

regina.fabry@rub.de

1 Department of Philosophy II, Ruhr University Bochum, Bochum, Germany 


\section{Introduction}

Since the 1980s, research in evolutionary biology (Flynn et al. 2013; Laland and O'Brien 2011; Laland et al. 2000; Odling-Smee and Laland 2011; West and King 1987) has shown that niche construction activities by human and other animal populations are a ubiquitous phenomenon. This research, and its theoretical implications, has received great attention in philosophy of biology (Sterelny 2003, 2012; Stotz 2010, 2017) and philosophy of cognition (Bertolotti and Magnani 2017; Clark 2005, 2006, 2008; Menary 2014, 2015; Sterelny 2010). Building on this research, with a particular appraisal of Stotz's (2017) work, I will argue that we should establish and maintain, on ontological and conceptual grounds, a distinction between selective niche construction, developmental niche construction, and organism-niche coordination dynamics, and that these notions capture different causal patterns in the physical world.

Recently, the insight that many cognitive phenomena are best understood by analysing the reciprocal causal relationship between organisms and their actively constructed environment has sparked interest in proponents of the free-energy principle and variational active inference, who seek to explain multiple mental phenomena as a continuous attempt to minimise free energy (see, e.g., Ramstead et al. 2019; Ramstead et al. 2016; Veissière et al. 2020). The overall goal of this paper is to explore the potential of the variational active inference scheme for deepening our understanding of selective niche construction, developmental niche construction, and organism-niche coordination dynamics. I will focus on the examination and interpretation of the extended active inference account developed by Constant et al. (2020), but I will also take the variational approach to niche construction by Constant et al. (2018) into consideration. I will discuss to what extent the theoretical integration of the variational active inference scheme (Friston 2013b; Hesp et al. 2019; Ramstead et al. 2018) and philosophical work on extended cognition (Clark 2008; Clark and Chalmers 1998; Kirchhoff 2012), proposed by Constant et al. (2020), succeeds in deepening our understanding of the reciprocal causal relationship between organisms and their niche.

I will proceed as follows. In a first step, I will specify the notions of selective niche construction, developmental niche construction, and organism-niche coordination dynamics (sections "Selective niche construction and developmental niche construction" and "Organism-niche coordination dynamics"). Building on this characterisation, I will argue that these notions capture distinct causal patterns and that each of them has a specific scope (Sect. "Identifying causal patterns"). In a second step, I will depict the free-energy principle and summarise the main tenets of the variational active inference scheme (Sect. "The free-energy principle and variational active inference"). Against this background, I will provide a critical analysis of the extended active inference account-and key aspects of the variational approach to niche construction (Sect. "Extended active inference: A critical analysis"). My key claim will be that the extended active inference account has the potential to deepen our understanding of organism-niche coordination dynamics, but misses out on the opportunity to adequately capture 
selective niche construction and developmental niche construction. Finally, this claim will inspire a close examination of the feasibility and implications of a theoretical integration of the variational active inference scheme with work on extended cognition (Sect. "Extended active inference and organism-niche coordination dynamics"). The upshot of this examination will be that this integration can indeed elucidate organism-coordination dynamics, but that more work needs to be done to move away from an organism-centred depiction of cognitive functions to capturing the dynamics of collective action and collaboration in the niche. In conclusion, I will suggest to limit the explanatory scope of the extended active inference account on formal and conceptual grounds. This conclusion comes with an invitation to the proponents of this account to elaborate on their formal and conceptual characterisation of organism-niche coordination dynamics and to show how the variational active inference scheme can elucidate selective niche construction and developmental niche construction.

\section{Selective niche construction, developmental niche construction, and organism-niche coordination dynamics}

Research in evolutionary biology (Flynn et al. 2013; Laland and O'Brien 2011; Laland et al. 2000; Odling-Smee and Laland 2011), philosophy of biology (Sterelny 2003, 2012; Stotz 2010, 2017), and philosophy of cognition (Bertolotti and Magnani 2017; Clark 2005, 2006, 2008; Menary 2014, 2015; Sterelny 2010) emphasises the crucial role of niche construction in shaping and transforming the selective fitness, life cycles, and cognitive abilities of humans and other animals. The basic assumption is that the active construction and re-construction of the local environment leads to the modification of population-specific phylogenetic and ontogenetic trajectories, as well as to genuinely new opportunities for individual action and cognition in the here-and-now. To a first approximation, niche construction " $[\ldots]$ refers to the activities, choices, and metabolic processes of organisms, through which they define, choose, modify, and partly create their own niches" (Laland et al. 2000, pp. 132-133; see also Flynn et al. 2013; Odling-Smee and Laland 2011). Importantly, the notion of 'niche construction' has been used to refer to ontologically distinct phenomena. It is therefore crucial to introduce and maintain a conceptual distinction between selective niche construction, developmental niche construction, and organism-niche coordination dynamics.

\section{Selective niche construction and developmental niche construction}

As Stotz (2017) has convincingly argued, it is important to distinguish between selective niche construction (SNC) and developmental niche construction (DNC) (see also Griffiths and Stotz 2018). The notion of selective niche construction refers to a reciprocal causal relationship between the collective, cross-generational modification of the local environment by a population of organisms and the changes of selection pressures upon that population induced by it in the course of evolution 
(Laland et al. 2000). In other words, "[t]he selective niche is defined by the environmental parameters which have a causal influence on the differential survival and reproduction rate of organisms" (Stotz 2017, p. 2), where these parameters are a direct result of active construction processes within a population across multiple generations. This specification of selective niche construction builds on niche construction theory in evolutionary biology (Laland et al. 2000), which suggests that populations of human and non-human animals causally influence the genotype of future generations through their active construction of the local environment (Flynn et al. 2013).

A key example of the causal impact of niche construction on the selection of adaptive traits is phenotypic plasticity. We should distinguish between two types of phenotypic plasticity, i.e., behavioural plasticity and developmental plasticity (Sterelny 2003; Stotz 2010). First, in response to and in interaction with variable and potentially unstable environments, where these variabilities and instabilities are in part induced by niche construction processes, organisms display new behavioural repertoires and action schemes. Second, developmental plasticity captures the potential to flexibly develop new skills and evaluation and appraisal mechanisms (Sterelny 2003, p. 163; for a discussion, see Clark 2008). Crucially, an important feature of human brains is learning-driven plasticity (Menary 2015), which enables the development of new functional and structural neuronal connections that are associated with specific cases of organism-niche coordination dynamics (see section "Organism-niche coordination dynamics"). Sterelny (2003) assumes that "[...] there is every reason to think that developmental plasticity is an adaptation", given that at least some of its features " $[. .$.$] enhance current fitness by equipping agents for the specific features of$ the environment" (p. 166; for a discussion, see Stotz 2010). Developmental plasticity can thus be seen as a selective response to niche construction and contributes, in important ways, to the variability and flexibility of human cognitive capacities.

This example also highlights the crucial ontological and conceptual difference between selective niche construction and developmental niche construction: while selective niche construction describes the changes of selection pressures caused by the population-specific modification of the environment, developmental niche construction refers to changes of the expression of phenotypic traits caused by the collective construction and re-construction of the environment (Stotz 2017). In other words, "[t]he constructed developmental niche captures the exogenetic (e.g. ecological and social) legacies an organism inherits alongside its genes that together ensure the-potentially modified-reconstruction of the life cycle of the next generation" (Stotz 2017, p. 2). The idea of developmental niche construction, Stotz (2017) shows, originates in West and King's (1987) conceptualisation of the ontogenetic niche, which is defined as "the set of ecological and social circumstances inherited by organisms" (p. 550).

For each generation of organisms, the developmental niche, understood as the outcome of the active inter-generational transmission of information and resources relevant for ontogenetic development, is "constructed in interaction with parents, other conspecifics, the physical and biological environment, and cognitive artefacts from tools to languages" (Stotz 2017, p. 3; see also Stotz 2010; Griffiths and Hochman 2015; Griffiths and Stotz 2018). Specifically, in the human case, the 
developmental niche is characterised by modes of cultural learning (Heyes 2012, 2018), institutions for cultural learning (e.g., kindergartens, schools), language, representational systems (e.g., writing systems, numerical systems), and cognitive tools (e.g., pen and paper, abaci, computers), and so on (cf. Menary and Gillett 2016). The members of each generation modify their own developmental niche and the developmental niche of the subsequent generation through their collective interaction with resources in their environment-they contribute to "cumulative downstream niche construction" (Sterelny 2003, p. 150; italics removed; see also Sterelny 2010, 2012). A particularly relevant type of human cumulative downstream niche construction that helps us understand the peculiarity of human cognition, Sterelny $(2003,2012)$ argues, is informational epistemic engineering: “[...] human cognitive competence often depends on epistemic engineering: on organizing our physical environment in ways that enhance our information-processing capacities" (Sterelny 2012, p. xii; for discussions, see Clark 2008; Stotz 2010). Through informational epistemic engineering, and other forms of developmental niche construction, populations and sub-populations of organisms thus change the environmental landscape they and the members of the next generation(s) are inhabiting, exploiting, and manipulating.

Stotz (2017) mentions two key functions of developmental niche construction: species-specific typicality and diversity. First, the characteristics of the developmental niche enable the "species-typical expression of the genetic factors" (Stotz 2017 , p. 4), thereby ensuring that all organisms within a species develop typical phenotypic traits. Second, developmental niche construction gives rise to phenotypic diversity across populations, because "the developmental niche also provides input to developmental plasticity" (ibid.). In other words, the expression of the adaptive trait of developmental plasticity, which likely is a product of selective niche construction (see above), depends upon the particular features of the environment, i.e., the population-specific results of developmental niche construction. This gives rise to the question as of how the effects of developmental niche construction can be characterised if we consider the coordination of individual organisms and their developmental niche. I will address this question in the next sub-section.

\section{Organism-niche coordination dynamics}

Following Stotz (2017), I have described developmental niche construction as a cumulative, inter-generational process that is realised by a population or sub-population of organisms. However, philosophers have also paid attention to the reciprocal causal interaction between an individual organism and its developmental niche in the here-and-now. I refer to these interactions as organism-niche coordination dynamics (cf. Menary 2013; Menary and Gillett 2016). These interactions of an individual organism and its developmental niche lead to the acquisition and application of collectively shaped, culturally evolved knowledge and skills (Sterelny 2003; Stotz 2010).

This is closely related, I suggest, to the way in which Clark (2008) captures the crucial contributions of the cognitive niche to cases of extended cognition. In its original formulation (Clark and Chalmers 1998), the thesis of extended cognition 
says that many cognitive processes (e.g., remembering, problem-solving) extend from the brain to the rest of the body and the local environment. Being opposed to internalism about cognitive functioning, the extended cognition thesis rests on the parity principle: "If, as we confronted some task, a part of the world functions as a process which, were it done in the head, we would have no hesitation in recognizing as part of the cognitive process, then that part of the world is (so we claim) part of the cognitive process" (Clark and Chalmers 1998, p. 8; italics in original). An extended cognitive function has to meet (to varying degrees) the following criteria of trust and glue: (1) the worldly or environmental resource that forms part of the cognitive process has to be "reliably available and typically invoked"; (2) the information it delivers has to be "automatically endorsed", that is, it need not be subject to deliberate scrutiny by the organism; (3) the resource has to be "easily accessible as and when required" (Clark 2010, p. 46; for a discussion of these criteria, see, e.g., Sutton 2010). If these trust and glue criteria are fulfilled, the organism and a certain resource in the environment form a causally coupled system that qualifies as an extended cognitive system (Clark and Chalmers 1998). Importantly, as Clark and Chalmers (1998) argue, "[...] the relevant external features are active, playing a crucial role in the here-and-now" (p. 9; italics in original). This active causal role of resources in or features of the environment is specified by Clark (2008) in his discussion of cognitive niche construction.

Discussing the cognitive role of manipulating objects in space by individual cognisers, Clark defines cognitive niche construction "[...] as the process by which animals build physical structures that transform problem spaces in ways that aid (or sometimes impede) thinking and reasoning about some target domain or domains" (Clark 2008, p. 62). Many, if not all cases of thinking and reasoning discussed by Clark and Chalmers (1998) and Clark (2008) are captured as processes that are realised by the individual organism (Sterelny 2010). This is suggested, for example, by Clark's (2008) assumption that cognitive niche construction has an important impact on "the fitness landscape for individual lifetime learning" (Clark 2008, p. 62 ; emphasis added). Individual cognitive processes, proponents of extended cognition argue, extend into the cognitive niche. The cognitive niche, I would add, has been shaped and modified, through the trans-generational, cumulative process of developmental niche construction, which has brought about new tools and resources for individual cognition. This is in line with Kim Sterelny's (2010) claim that "[t]he most critical, mind-and-brain shaping environmental supports for cognition are these cumulatively built, collectively provided tools for thinking, tools that are provided to many or all of a generation by many or all of the previous generation" (p. 478; see also Menary 2015).

The important role of organism-niche coordination dynamics, which is distinct from developmental niche construction, is also emphasised in recent philosophical work on cognitive integration and enculturation (Menary 2013, 2015; Menary and Gillett 2016). The key assumption of the cognitive integration framework is that many, if not all culturally evolved, socio-culturally shaped cognitive processes are realised by "[...] multiple cognitive layers where neural, bodily, and environmental processes all conspire to complete cognitive tasks" (Menary 2015, p. 2). The notion of enculturation captures the transformation of individual cognitive 
capacities through the socio-culturally scaffolded acquisition of culturally evolved cognitive practices in the developmental niche. Cognitive practices can be defined as the embodied, socio-culturally shaped interaction of an organism with cognitive resources in the developmental cognitive niche (Menary 2015); key examples of cognitive practices include reading (Fabry 2018a; Menary 2014), writing (Fabry 2018b; Menary 2007), and arithmetic (Fabry 2020; Jones 2020; Menary 2015; Pantsar 2019). In the course of enculturation, individual organisms acquire, if all goes well, competence in cognitive practices - they undergo an active process of cognitive transformation. According to Menary (2015), these "[...] cognitive transformations occur when the development of the cognitive capacities of an individual are sculpted by the cultural and social niche of that individual" (p. 8). This suggests that cognitive practices are primarily realised by the coordination dynamics linking individual organisms and the developmental cognitive niche. It should be noted, however, that Menary (2013, 2015) acknowledges that cognitive practices are often characterised by the collaboration of several enculturated organisms who jointly complete a cognitive task. While cognitive integrationists emphasise the importance of selective niche construction and developmental niche construction for understanding the population-specific unfolding of cognitive practices across time, their analysis of integrated thinking, reasoning, and problem-solving focuses on "the physical dynamics of brain-body-niche interactions in real-time" (Menary and Gillett 2016, p. 73; emphasis added). The upshot is that the developmental cognitive niche plays a double role: it makes crucial causal contributions to cognitive transformation, and it provides the individual organism with resources and opportunities for the (collaborative) completion of cognitive tasks.

\section{Identifying causal patterns}

Selective niche construction, developmental niche construction, and organismniche coordination dynamics contribute to a single, pervasive phenomenon: the reciprocal relationship between organisms and their environment. However, building on recent work in philosophy of science (Potochnik 2017; Potochnik and Sanches de Oliveira 2020), I argue that the three concepts capture different causal patterns. Causal patterns are defined as "regularities in phenomena" (Potochnik 2017, p. 25), which feature as "explanatory dependence relations" in scientific explanations (ibid., p. 140). Given that we are living in a causally complex world, "any given phenomenon embodies many causal patterns" (ibid., p. 200). Across scientific contexts, causal patterns serve the purpose of identifying causal dependence relations that can partly explain the occurrence of the target phenomenon. Furthermore, "[...] they indicate the scope of that dependence" (p. 139; italics in original; see also Potochnik and Sanches de Oliveira 2020). The general aim of causal scientific explanation, then, is to represent a causal pattern and to show how it contributes, perhaps also relative to other causal patterns, to the manifestation of the phenomenon under investigation. A consequence of causal complexity and the multiplicity of causal patterns is that "[t]he same phenomenon may $[. .$.$] be targeted by many different research programs, in principle$ 
or in fact" (Potochnik 2017, p. 200). Following up on this idea, Potochnik and Sanches de Oliveira (2020) suggest that scientific explanation in the cognitive sciences (and well beyond) requires a "pluralism of explanatory style" (p. 9; italics removed). Each explanatory style, understood as a practice-based mode of gaining knowledge through scientific explanation, is in the service of identifying, representing, and understanding a relevant causal pattern and its scope.

In sum, the suggestion is that a target phenomenon is characterised by diverse causal patterns with a certain scope and that these dependence relations need to be captured by employing different explanatory styles. This presents an alternative to the assumption that phenomena have a hierarchical, compositional structure and that this structure warrants a distinction of different levels of organisation and different, corresponding levels of explanation (Oppenheim and Putnam 1958; for a discussion, see Craver 2015). Several philosophers of science have convincingly argued that the postulation of a hierarchical organisation of the physical world is problematic on ontological and epistemological grounds (DiFrisco 2017; Eronen 2015; Potochnik and McGill 2012). The general assumption that a state or process at level $l$ is composed solely of states and processes at level $l-l$ has been falsified by a number of examples showing that causal dependence relations explaining the occurrence of the relevant target phenomenon cut across organisational levels (Potochnik and McGill 2012). This calls the explanatory relevance and feasibility of the postulation of the hierarchical organisation of the physical world into question. Engaging in detail with this argument is beyond the scope of the present paper. What matters for current purposes is the proposal that the dependence relations holding for a certain target phenomenon should be captured by different causal patterns, which require us to employ adequate explanatory styles, rather than assuming that dependence relations can be ascribed to discrete levels of organisation on corresponding levels of explanation (Potochnik 2017; Potochnik and Sanches de Oliveira 2020).

The idea that a target phenomenon is characterised by multiple causal patterns, each with a specific scope, helps us specify the distinction between selective niche construction, developmental niche construction, and organism-niche coordination dynamics. Selective niche construction captures the modification of selection pressures that results from the niche construction activities by an entire population across multiple generations. By contrast, developmental niche construction is characterised by the modification of phenotypic traits that is a direct outcome of niche construction activities by an entire population (or sub-population) across at least two generations. Given these differences, the causal patterns are clearly distinct, while it remains a theoretical option that " $[\mathrm{t}]$ he developmental and selective niche of a population can but do not have to overlap" (Stotz 2017, p. 2).

Based on Stotz's (2017) analysis, there are at least two causal aspects that clearly distinguish selective niche construction and developmental niche construction. First, while selective niche construction captures changes to the genotype, developmental niche construction captures changes to the phenotype. Second, both types of niche construction have a different temporal scope. Selective niche construction is a process that spans multiple generations across evolutionary time. Developmental niche construction, however, is a process that is realised by atleast two generations. Both 
selective niche construction and developmental niche construction are processes that are realised by multiple organisms within a population.

By contrast, organism-niche coordination dynamics captures the reciprocal causal dependence relation between an individual organism and its (cognitive) niche. The temporal scope of this causal pattern is far more limited than the temporal scope of both selective and developmental niche construction. The dynamical coordination of an individual organism and its niche is realised in the here-and-now. This still allows for the option to identify task-specific coordination dynamics at time points $t_{1}, t_{2}, t_{3}, \ldots$, for example if we aim for a specification of the learning trajectory of an individual organism in interaction with its niche.

In sum, selective niche construction, developmental niche construction, and organism-niche coordination dynamics are ontologically and conceptually distinct. This distinction can be specified by taking the relevant causal pattern, the quantity of causally relevant organisms, and the temporal scope of the dependence relation into consideration. ${ }^{1}$

\section{The free-energy principle and variational active inference}

Recently, proponents of a variational approach to human cognition have argued that the reciprocal causal relationship between an organism and its niche can be captured by describing it formally and conceptually as a process of free energy minimisation (Bruineberg et al. 2018; Constant et al. 2018, 2020). According to the free-energy principle (FEP), "[...] any self-organising system that is at equilibrium with its environment must minimize its free energy" (Friston 2010, p. 127). The FEP can be described as a "first-principle" (Colombo and Wright 2018), which can help us understand, its proponents argue, the structural and functional processes that characterise living biological systems (Friston 2013b). For current purposes, I will focus on the implications of the FEP and Variational Active Inference, ${ }^{2}$ one of the process theories building on it (Hohwy 2020), for our understanding of selective niche construction, developmental niche construction, and organism-niche coordination dynamics.

In order to maintain its physical integrity in an environment that is characterised by multiple variable and fluctuating states, any organism must minimise the entropy of its sensory states (Friston 2013b). The minimisation of entropy entails that "[...]

\footnotetext{
1 It should be noted that Bertolotti and Magnani (2017) seem to pursue a similar strategy. While they do not distinguish between selective niche construction and developmental niche construction, they do maintain that there is a difference between ecological niche construction (equivalent to selective niche construction) and cognitive niche construction (equivalent to organism-niche coordination dynamics) and that these distinct causal patterns should be explained in different ways. While the ecological niche and its constructors stand in an enabling relationship, cognitive niche construction, Bertolotti and Magnani (2017) argue, can be described in relational terms by assuming that individual cognisers modify the affordances available to them.

2 In what follows, I will refer to the process of free energy minimisation as 'variational active inference' and to the theoretical account describing this process mathematically and conceptually as 'Variational Active Inference' in capital letters.
} 
there is a high probability that a system will be in any small number of states, and low probability that it will be in the remaining states" (Friston 2010, p. 127). Formally, entropy reflects the long-term average of surprisal, which is defined as the negative log probability of an organism's sensory states (Friston 2010, 2013b). Organisms cannot assess and minimise surprisal directly, given that surprisal could depend, in principle, on multiple hidden causes in the environment to which the organism does not have direct access (Hohwy 2015). The computational intractability of directly minimising surprisal forces free energy, understood as an informationtheoretic quantity, into the picture: "[...] free energy is an upper bound on surprise, which means that if agents minimize free energy, they implicitly minimize surprise" (Friston 2010, p. 128). ${ }^{3}$ Free energy is minimised through adaptive behaviour given a probabilistic generative model. The model is generative, because its purpose is "[...] to capture the statistical structure of some set of observed inputs by inferring a causal matrix able to give rise to that very structure" (Clark 2016, p. 21). The model is probabilistic, because it captures the causal structure of the environment in a way that approximates Bayesian variational inference (Hohwy 2015). For current purposes, it is important to clearly distinguish between a generative model and a generative process (Bruineberg et al. 2018). According to Constant et al. (2018), "[a] generative model is a mapping from sensory observations, action policies, to external (hidden) causes. These causes constitute the generative process, which describes the transitions among hidden causes in the world (including the organism's own actions) that generate sensory inputs" (p. 3; italics in original). In the present context, the generative process translates into the state transitions in the niche.

The internal states of an organismic generative model and external states that comprise the generative process are statistically separated by a Markov blanket (Friston 2013b; Friston et al. 2014). The Markov blanket is constituted by sensory states and active states. According to Friston (2013b), the distinction between internal and external states, which is induced by a Markov blanket, and the distinction between sensory and active states within the Markov blanket can be defined in the following way: "External states cause sensory states that influence - but are not influenced by - internal states, while internal states cause active states that influence - but are not influenced by - external states" (p. 2). The formal postulation of a Markov blanket, its proponents argue, allows for a specification of the reciprocal causal relationship between an organismic generative model and a generative process (i.e., state transitions in the niche).

An organism that is situated in a niche minimises its free energy by optimising its generative model through variational active inference. The generative model encodes priors (i.e. Bayesian beliefs) about the most probable causes of sensory input. These priors are modified in light of new sensory evidence, which corresponds to perception, and induce changes to sensory input through sensory sampling, which corresponds to action (Constant et al. 2018, 2020; Hesp et al. 2019). The continuous

\footnotetext{
${ }^{3}$ In the literature on the FEP, surprisal is sometimes also called surprise. Following Clark (2013), in order to avoid the misunderstanding that this information-theoretical quantity is equal to the phenomenal. experience of being surprised, I prefer to use the more technical term surprisal.
} 
updating of the generative model, if all goes well, leads to the minimisation of free energy and therefore to the minimisation of surprisal.

Under variational active inference, the relative causal influence of perception and action on free energy minimisation is modulated by the estimated precision (inverse variance) of sensory signals (Friston 2010; Veissière et al. 2020). This continuous process of "second order statistics" assesses the certainty in sensory signals and therefore the strength of their assigned causal influence on the updating of prior beliefs (Hohwy 2013, p. 66).

Importantly, salient aspects of the environment promise to provide precise sensory signals for perception, thereby contributing to the efficiency of free energy minimisation (Veissière et al. 2020). Salience, in this context, is defined as "[...] the information gain, reduction of uncertainty, Bayesian surprise or epistemic value" (Constant et al. 2018, p. 9), where epistemic value denotes "the expected information gain under predicted outcomes" (Friston et al. 2015, p. 192). Salience, then, is a property of external states relative to the internal states of an organism. What emerges is a variational description of a perceiving and acting organism that optimises its generative model of external states in the niche in virtue of the statistical postulation of a Markov blanket.

\section{Extended active inference: $A$ critical analysis}

Building on the FEP, the postulation of Markov blankets, and the formal and conceptual description of variational active inference, the proponents of the extended active inference account, on which I will focus in this paper, suggest that it could "[...] provide a mechanistic basis for the psychological and functional aspects of the cognitive niche" (Constant et al. 2020, p. 9). Similarly, the closely related variational approach to niche construction, its proponents argue, "[...] could provide a promising modelling tool for research on the niche construction perspective" (Constant et al. 2018, p. 2). The epistemic goal of the extended active inference account and the variational approach to niche construction is, therefore, to capture the reciprocal causal relationship between an organism and its niche.

Referring to Stotz (2017) and West and King (1987), Constant et al. (2020) introduce a distinction between selective niche construction, developmental niche construction, and cognitive niche construction (equivalent to organism-niche coordination dynamics). However, they conflate this ontological and conceptual distinction: "The concept of the cognitive niche we refer to here is a sort of hybrid between the concepts of the selective, developmental, and cognitive niches" (p. 3). Unfortunately, they do not provide any reasons for this conflation. Similarly, in introducing their variational approach to niche construction, Constant et al. (2018) build on Stotz (2017) to establish a distinction between selective niche construction and developmental niche construction. However, they seem to misinterpret Stotz's (2017) distinction by suggesting that "[the] developmental niche [...] [is] a temporal subset of the selective niche" (Constant et al. 2018, p. 2), rather than a distinct causal pattern, that may or may not overlap with the causal pattern captured by selective niche construction. Furthermore, it remains unclear how exactly this distinction thus 
established translates into their variational model. Their expectation that " $[\ldots]$ the DNC-SNC [developmental niche construction-selective niche construction] tandem [...] tend[s], over time and on average, to consolidate organism-niche complementarity across timescales" suggests that their model ultimately seems to conflate the ontologically and conceptually distinct processes of selective niche construction and developmental niche construction (Constant et al. 2018, p. 11). Given that selective niche construction, developmental niche construction, and organism-niche coordination dynamics capture different causal patterns with a different scope of the relevant dependence relations (see Sect. "Identifying causal patterns"), it is open to debate whether the extended active inference account and, to a lesser degree, the variational approach to niche construction can succeed in reaching their goal of describing the reciprocal relationship between organisms and their environment across scales. In what follows, I will focus on an analysis of the extended active inference account and enrich my observations and assumptions by taking the variational approach to niche construction into consideration.

Constant et al. (2020) are interested in the question as of how Variational Active Inference can contribute to a better understanding of "the computational function of cognitive extensions, and the (developmental and intergenerational) process whereby this computational function emerges" (p. 2). In exploring the relationship between an organism and its niche, they distinguish between the psychological habitat, defined as a space of action possibilities provided by the niche (i.e., affordances), and the functional habitat, defined as a space that provides epistemic resources for the completion of cognitive tasks. While the psychological habitat is set to explain the organism's phenomenal experiences that are associated with its interaction with the niche, the explanatory role of the functional habitat is to elucidate the causal contributions of the niche to an organism's ability to complete certain cognitive tasks. It is the functional habitat, understood as a sub-set of the overall physical habitat of organisms, that is key to understanding the functional role of the niche.

The reciprocal causal relationship between an organism and its niche, Constant et al. (2020) argue, can be aptly described in terms of variational active inference: "Changes in brain states and functional architectures optimize organisms' generative (i.e., causal) model of the causal structure of their cognitive niche" in moments of perception (p. 9), thereby minimising free energy. Using the mathematical tools of variational calculus, this optimisation of the generative model through approximate Bayesian belief updating is formalised in the following way (Constant et al. 2020, p. 7):

$$
F(s, \mu)=D\left[q_{\mu}(\eta) \| p(\eta \mid s)\right]-\ln p(s)
$$

$F(s, \mu)$ represents the free energy functional (the function of a function) of sensations $s$ and internal states $\mu$ of an organism, $D$ represents the Kullback-Leibler divergence measuring the divergence between the variational density $q_{\mu}(\eta)$ and the distribution of the posterior probability of sensory causes $p(\eta \mid s)$, which is always $\geq 0$. The value of surprisal is expressed by $-\ln p(s)$. This formalism captures the optimisation of an organism's generative model in perception. 
Conceptually, the optimisation of the model is modulated by the estimated precision of sensory signals. Precision estimation is understood as a "meta-learning mechanism" that facilitates the organism's "attunement to causal regularities in the environment" (Constant et al. 2018, p. 9). Precision estimation is therefore crucial for optimising the bound on surprisal.

Importantly, while perception can optimise the bound on surprisal, only action can minimise the bound on surprisal given that it is "an attribute of sensations actively sampled" (Friston 2013a, p. 212). In active inference, proprioceptive and interoceptive changes "[...] resolve uncertainty through the active sampling of salient, uncertainty reducing sensations, while allowing for preferred, unsurprising outcomes" (Constant et al. 2020, p. 8; see also Hesp et al. 2019). This is captured by the following formal expression (ibid.):

$$
F(s, \mu)=D\left[q_{\mu}(\eta) \| p(\eta)\right]-E_{q}[\ln p(s(a) \mid \eta)]
$$

Action, this expression suggests, minimises surprisal by sampling the niche in such a way that it conforms to expected sensory and internal states. Active sampling is guided by the salience of specific causal regularities in the niche. The salience of certain objects and artefacts, for example, structures the active minimisation of free energy (Constant et al. 2018). Salience, then, is a relational property establishing connections between the organismic generative model and components of the generative process, i.e., the niche. The salience of causal regularities in the niche is, in many cases, a result of previous niche construction activities. Constant et al. (2018, 2020) describe the process of actively creating salient causal regularities so as to facilitate free energy minimisation as uploading.

In the context of analysing cognitive cases of free energy minimisation, Constant et al. (2020) suggest that the notion of cognitive uploading is an extension of the concept of cognitive offloading, where cognitive offloading is defined as "the use of physical action to alter the information requirements of a task so as to reduce cognitive demand" (Risko and Gilbert 2016, p. 676). Offloading refers to the manipulation of objects in the niche by an individual organism so as to complete a specific cognitive task (e.g., remembering, problem-solving). In cases of uploading, Constant et al. (2020) propose, "[a] function is uploaded when social and technological change means it is now taken care of by the niche rather than the individual" (p. 5). I will return to the relevance of uploading for understanding variational active inference in the Sect. "Extended active inference and organism-niche coordination dynamics". For now, it suffices to note that salient causal regularities are, at least in many cases, the result of cognitive uploading. In turn, as Constant et al. (2020) argue, "[...] cognitive uploading helps agents to minimize the bound on surprisal" (p. 15).

So far, I have summarised Constant et al. (2020) conceptual and formal description of the ways in which an organism minimises its free energy by generatively modelling its niche (i.e., the generative process) in perception and action. As seen in previous section, free energy minimisation is dependent upon the statistical separation between internal and external states by a Markov blanket. The Markov blanket induces a formal symmetry between the internal states of the organism and the 
external states of the niche. Given this formal symmetry, it is possible to transform the variational formalism such that the niche becomes the generative model and the organism becomes the generative process (Constant et al. 2018, 2020). In other words, "[...] there must be a description of niche dynamics, where the environment models how the agent's internal dynamics are generating its active states" (Constant et al. 2018, p. 5). The consequence of this transformation is that the organism's active states are represented as the sensory states of the niche, while the sensory states of the organism become the active states of the niche (cf. ibid.). This circular causality (Bruineberg et al. 2018) of the states of the organism and its niche can be expressed by rewriting Eq. (1) so that the sensations $s$ and internal states $\mu$ of the organism in Eq. (1) become the organism's actions $a$ as 'sensed' by the niche and the states of the niche $\eta$ (Constant et al. 2020, p. 10):

$$
F(a, \eta)=D\left[q_{\eta}(\mu) \| p(\mu \mid a)\right]-\ln p(a)
$$

This formal transformation enables a description of the causal relationship between an organism and its niche 'from the perspective' of the niche. More importantly, however, it allows us to describe the organism and its niche as a causally coupled system that jointly minimises free energy. As Constant et al. (2018) put it, "[...] the generative model of both agent and niche are the veridical explanations of their respective sensory fluctuations and the variational free energies of both are jointly minimised with respect to the posterior beliefs parametrized by their respective states" (p. 7).

The assumption that the reciprocal causal relationship between an organism and its niche can be described in terms of joint free energy minimisation finds support in a recent simulation study of desire path behaviour, where this behaviour is a simplified example of niche construction (Bruineberg et al. 2018). In a nutshell, these simulations show how an organism and its niche (a simple maze) are mutually attuned to each other through the organism's actions (the carving of a path through the maze), which feed back into the organism's sensations of the niche, thereby jointly minimising free energy.

On this view, free energy minimisation is conceptualised as a process that comprises the coupled organism-niche system. In this sense, Constant et al. (2020) argue, free energy minimisation is extended: "[...] the free energy of two systems (i.e., organism and niche) is just the sum of their respective free energies, conditioned upon the (i.e., sensory and active) states they share (Bruineberg et al. 2018)" (p. 12). Under this description, active inference extends into the niche. The upshot of Constant et al. (2020) account is a theoretical integration of Variational Active Inference and philosophical work on extended cognition (Clark 2008; Clark and Chalmers 1998; Kirchhoff 2012, 2015). I will discuss the feasibility and implications of this integration in detail in the next section.

For now, the question arises how the extended active inference account relates to the distinction, introduced above, between selective niche construction, developmental niche construction, and organism-niche coordination dynamics. These three concepts, I have argued above, capture different causal patterns with a distinct scope. Recall that Constant et al. (2020) state that "[t]he concept of the 
cognitive niche we refer to here is a sort of hybrid between the concepts of the selective, developmental, and cognitive niches" (p. 2). This conceptual hybridisation, however, conflates important ontological and conceptual differences between selective niche construction, developmental niche construction, and organism-niche coordination dynamics. This conflation would render the variational model of the reciprocal causal relationship between an organism and its niche unconvincing, as this model would not capture the different causal patterns - and their inter-relations - characterising the target phenomenon. This conclusion, however, could be avoided if we re-assess the niche conception that is at the core of the extended active inference account. It is possible, I propose, to appreciate the explanatory merits of the extended active inference account without committing to the conceptual conflation of selective niche construction, developmental niche construction, and organism-niche coordination dynamics.

To explore this possibility, I will take a closer look at the variational model suggested by Constant et al. (2020) and interpret it against the background of the distinction between the different causal patterns that contribute to the target phenomenon, i.e., to the reciprocal relationship between organisms and their environment. The formal description of extended active inference, I argue, represents the causal reciprocal relationship between an individual organism and its cognitive niche. Equations (1) and (2) above have it that it is the individual sensing and acting organism that generatively models the niche. Given the formal symmetry between the internal states of the organism and the external states of the niche induced by the Markov blanket, the states that realise extended active inference, captured in Eq. (3), are, likewise, the states of an individual organism and its cognitive niche. This joint minimisation of free energy, my interpretation of the formal expression of extended active inference implies, is realised in the hereand-now or at times $t_{1}, t_{2}, t_{3}, \ldots$, if we wish to describe the temporal unfolding of extended active inference. As it stands, the extended active inference account does not capture selective niche construction, for it remains silent on the selection pressures induced by niche construction activities by an entire population across multiple generations. Furthermore, the extended active inference account does not capture developmental niche construction, because its focus is on the individual organism, rather than on an entire population (or sub-population) of organisms that modifies the cognitive niche across two (or more) generations, thereby changing the array of possible phenotypic traits. Contrary to the stipulation by Constant et al. (2020), then, extended active inference does not capture "a sort of hybrid between the concepts of the selective, developmental, and cognitive niches" (p. 2), but organism-niche coordination dynamics.

This conclusion can be further substantiated by taking the assumptions of the original extended cognition thesis into consideration. Recall that the analysis of cognitive niche construction developed by Clark (2008) focuses on the engagement of an individual organism with resources in the cognitive niche (see Sterelny 2010). Furthermore, in their original proposal of the extended cognition thesis, Clark and Chalmers (1998) stress that the cognitive resources in the niche "[...] are active, playing a crucial role in the here-and-now" (p. 9; italics in original). This way of theorising identifies the individual organism in its occurrent interaction with the 
cognitive niche as the relevant causal pattern for explaining cognition. Given that the extended active inference account is committed to these key principles of extended cognition, it follows that this account captures organism-niche coordination dynamics, rather than selective niche construction or developmental niche construction.

A similar interpretation of the explanatory scope of Variational Active Inference applies, I suggest, to Constant's et al. (2018) variational approach to niche construction. ${ }^{4}$ Their formal model of the reciprocal causal organism-niche relationship, and joint free energy minimisation, bears a strong resemblance to the model developed by the extended active inference account. Their model, it seems to me, captures organism-environment coordination dynamics, rather than selective niche construction or developmental niche construction. This becomes obvious in their analysis of situated learning under variational active inference, for example, which provides a formal and conceptual interpretation of the ways in which an organismic generative model is updated so as to be optimally attuned to the generative process, i.e., the niche. According to Constant et al. (2018), situated learning

[...] requires of organisms that they engage conventionalized patterns of collective activity, grounded in material artefacts and other physical aspects of the niche, which have been transmitted to allow for the progressive integration of the organism as a legitimate member of the community. [...] By providing opportunities for learning how to learn, the developmental niche does not merely indicate what information ought to be learned, but also how best to learn it. As a meta-learning device, the information encoded in the material states of the local environment function to guide active inference by weighting sensory inputs according to their reliability, or salience, and constitute a more general device that allow organisms to learn how to learn, by guiding actionperception cycles (active inference). (p. 9)

Clearly, active inference, precision estimation, and the active exploitation of salient causal regularities in the niche, as they are described by Constant et al. (2018), are features of organism-niche coordination dynamics. The reason for this is that "action-perception cycles", precision estimation, and salience exploitation are realised by an individual organism, rather than by a population of organisms, in the here-and-now and across time points $\mathrm{t}_{1}, \mathrm{t}_{2}, \mathrm{t}_{3}, \ldots$, not across the lifetimes of multiple generations (in the case of selective niche construction) or across the lifetimes of at least two generations (in the case of developmental niche construction), as I have argued above. The developmental niche certainly plays a role in organismniche coordination dynamics, and this role is captured by Constant's et al. (2018) treatment. However, what is not captured by this approach is the population-specific, trans-generational, cumulative process of developmental niche construction, even though Constant et al. (2018) might claim otherwise. Furthermore, the variational model suggested by Constant et al. (2018) does not represent the causal pattern of selective niche construction.

\footnotetext{
4 This interpretation also applies to the variational ecology framework (Ramstead et al., 2019), which recapitulates and integrates the variational approach to niche construction (Constant et al., 2018).
} 
My claim that the variational model, as it stands, exclusively captures organismniche coordination dynamics, and leaves the causal patterns of selective niche construction and developmental niche construction unexplained, can be further substantiated by noting how Constant et al. (2018) summarise their approach:

Active inference allows the organism to specify (often implicitly) those features of the environment that will be adaptive given the demands of their phenotype, i.e. they can specify those features of the niche, the learning of which makes them an accurate model of their environment. On a developmental timescale, this rests on the meta-learning function of niche construction, which guides optimization, or phenotypic plasticity. On the scale of phylogeny, it rests on the inheritance of constraints passed on across generations in the form of species relevant information (salience), which will further guide the optimization in development. (p. 11; emphasis added)

I take it as uncontroversial that organism-niche coordination dynamics, which is captured by the variational model, "rests on" population-specific, cross-generational processes of selective niche construction and developmental niche construction. Yet, I do maintain that organism-niche coordination dynamics, selective niche construction, and developmental niche construction are characterised by different causal patterns, each with a different scope.

The upshot is that the extended active inference account (Constant et al. 2020) and the variational approach to niche construction (Constant et al. 2018) provide variational models of organism-niche coordination dynamics. However, these models do not represent the causal patterns at play in selective niche construction and developmental niche construction.

In reply to this conclusion, the proponents of the extended active inference account (Constant et al. 2020) and the variational approach to niche construction (Constant et al. 2018) have three options. First, they could deny that there is a nontrivial ontological and conceptual distinction between selective niche construction, developmental niche construction, and organism-niche coordination dynamics. They would then have to show why this distinction is not relevant for their variational models and why and how the extended active inference account and the variational approach to niche construction do capture all three causal patterns.

Second, they could (at least to a certain extent) agree with my analysis and suggest that the reliance of their models on the Markov blanket formalism allows them to extend their model so as to capture selective niche construction and developmental niche construction as I have characterised it. According to variational neuroethology (Ramstead et al. 2019), a recent framework based on Variational Active Inference, the general definition and formalisation of Markov blankets allows for the possibility of recursively nested Markov blankets. The idea is that "[...] the Markov blanket ontology can be reiterated recursively, such that the MBs [Markov blankets] at any one scale are composed in turn of MBs at the scale above and below - which are also made of MBs, and so on, all the way up and all the way down" (Ramstead et al. 2019, p. 192; see also Hesp et al. 2019). They could then attempt to show, on formal and conceptual levels, that selective niche construction and developmental niche construction could be captured by modelling them as processes defined 
over different ensembles of recursively nested Markov blankets. This strategy, if the resulting formalisations were mathematically and computationally tractable, could save the general idea that Variational Active Inference has the resources to capture selective niche construction, developmental niche construction, and organism-niche coordination dynamics while avoiding the limitations of the current versions of the extended active inference account and the variational approach to niche construction.

Finally, a third option for replying to the conclusion of my analysis would be to agree that the extended active inference account and the variational approach to niche construction only capture organism-niche coordination dynamics and allow for the possibility that different explanatory styles might be needed for understanding selective niche construction and developmental niche construction, for example non-variational mathematical models in evolutionary biology (Laland et al. 2000) and developmental systems theory (Griffiths and Hochman 2015; Griffiths and Stotz 2018), respectively. This would entail a commitment to "pluralism of explanatory styles" (Potochnik and Sanches de Oliveira 2020, p. 1314).

These three options offer a standing invitation to the proponents of the extended active inference account and the variational approach to niche construction to reply to my analysis. For the time being, however, I submit that the extended active inference account, against the key assumption made by its proponents, merely captures organism-niche coordination dynamics and leaves the question as of how selective niche construction and developmental niche construction can be explained in terms of variational active inference unresolved. Still, this allows for the possibility to examine the integration of Variational Active Inference with philosophical theorising about extended cognition. I will take up this task in the next section.

\section{Extended active inference and organism-niche coordination dynamics}

Recall that the extended cognition thesis, as originally formulated by Clark and Chalmers (1998), rests on the parity principle. This principle would be confirmed, Constant et al. (2020) argue, by the assumption that "[...] the description of the dynamics underlying learning in the generative process are formally equivalent to the learning in the generative model" (p. 16). In other words, formal equivalence implies functional parity of internal and external states. The parity principle, as Sutton (2010) notes, emphasises the functional isomorphism of internal and external states. According to the extended active inference account, internal and external states are functionally isomorphic, at least at a coarse-grained level, given the formal symmetry of the individual organism and its niche, which is statistically induced by the Markov blanket (Constant et al. 2020).

This formal reframing of the parity principle and the (coarse-grained) functional isomorphism of internal and external states, it seems to me, forces a recapitulation of the discussion of the theoretical merits of the parity principle for understanding paradigm cases of extended cognition into the picture. Under the assumption that organism-niche interactions play, in many cases, an indispensable role in the completion of cognitive tasks, Menary (2010a, b) and Sutton (2010) have argued that a 
complementarity principle should be preferred over the parity principle to describe the reciprocal causal relationship between an organism and its cognitive niche. Sutton (2010) defines the complementarity principle in the following way (p. 194):

[...] in extended cognitive systems, external states and processes need not mimic or replicate the formats, dynamics, or functions of inner states and processes. Rather, different components of the overall (enduring or temporary) system can play quite different roles and have different properties while coupling in collective and complementary contributions to flexible thinking and acting.

External states in the niche, this definition of the complementarity principle suggests, do not play a crucial role in cognising because they are functionally isomorphic to organismic internal states, but because they have different properties and thereby complement the properties of organismic states. In other words, "[...] it is because the external vehicles provide a different kind of functionality and because they can coordinate with internal processes that they are integral parts of our cognitive systems" (Menary 2010a, b, p. 235). The complementarity principle, then, motivates the cognitive integration framework (Menary 2013, 2015; Menary and Gillett 2016), briefly described above, and its commitment to organism-niche coordination dynamics for understanding the manifestation of (socio-culturally shaped) cognitive capacities.

The complementarity principle, it seems to me, is fully consistent with extended active inference, as it captures, on a conceptual level, the mutual attunement of an individual organism and its cognitive niche in the business of jointly minimising free energy (and thereby surprisal). In support of this assumption, it should be noted that Constant et al. (2018) emphasise the importance of "organism-niche complementarity" for understanding their reciprocal causal relationship expressed by the formalisation of variational active inference (p. 10). Furthermore, the formal symmetry of internal and external states induced by the statistical properties of a Markov blanket does not entail functional parity.

Perhaps, then, it would be preferable to rely on the complementarity principle, rather than on the parity principle, to fully appreciate the crucial role of the niche for cognitive functioning. The complementarity principle invites us to consider how external resources in the cognitive niche often transform the overall cognitive processing capacities of the individual organism. The cognitive integration framework and its account of enculturation, for example, makes a strong case for the cognitive transformation of individual organisms by virtue of their active engagement with external resources, e.g., linguistic and numerical symbols, in the cognitive niche (Menary 2007, 2014, 2015). For current purposes, however, it is important to note that Clark (2006), refining the original extended cognition thesis, capitalises on the importance of the complementarity of internal organismic states and the properties of external states in the niche for understanding the crucial role of oral and written language for our cognitive processing capacities: "Central to this vision of language as a complementary resource is an appreciation of the power of added worldly structure (in this case, perceptible material symbols) to transform the tasks that confront an intelligent agent" (Clark 2006, p. 371; my emphasis) The transformative "power" 
of linguistic symbols and other resources in the cognitive niche, I suggest, is rendered possible by their complementarity to, rather than the functional isomorphism with, the internal states of the organism. The complementarity principle, then, would be entirely consistent with the minimisation of free energy by joint organism-niche systems as described by the extended active inference account. In contrast to the parity principle, it has the advantage of highlighting the importance of the unique properties of external resources in the niche for understanding their substantial contributions to cognitive extension.

This examination of the conceptual relationship between the parity principle and the functional isomorphism of internal and external states on the one hand and extended active inference on the other hand invites us to examine Constant et al. (2020) conception of cognitive uploading, already introduced in the previous section, in more detail. According to this conception, "[...] the notion of uploading refers to the creation of novel cognitive functions that are taken on board by the cognitive niche per se, instead of being merely managed by the cognitive niche" (Constant et al. 2020 p. 5; see also Constant et al. 2018). This notion helps specify, the proponents of the extended active inference account suggest, "[...] individualand group-level dynamics unfolding over developmental and intergenerational time scales" (ibid.). Against the backdrop of a clear distinction between selective niche construction, developmental niche construction, and organism-niche coordination dynamics, this conception of uploading seems to characterise an important aspect of developmental niche construction, thereby capturing a causal pattern that is distinct from organism-niche coordination dynamics, which is, I have argued, the causal pattern targeted by the extended active inference account.

Constant et al. (2020) consider another aspect of cognitive uploading, stating that "[n]ovel cognitive functions produced through cognitive uploading can result from gene-culture coevolutionary dynamics that 'glue' organisms to those functions performed by the 'trusted' niche" (ibid.). This way of characterising cognitive uploading seems to capture the causal pattern of selective niche construction, given that it forces the impact of niche construction activities on genetic selection pressures into the picture. It seems, then, that cognitive uploading is a process that needs to be ascribed to selective niche construction and developmental niche construction.

The question arises why the trust and glue criteria, established by Clark and Chalmers (1998) and Clark (2010) to specify the extendedness of cognitive functions, are applied to cases of extended active inference. The trust and glue criteria, I assume, were originally established for providing a specification of the causal pattern of organism-niche coordination dynamics as described by the extended cognition thesis (see Sect. "Organism-niche coordination dynamics"). Thus, these criteria, which specify, in the present context, that external states ascribed to a resource in the cognitive niche have to be "reliably available and typically invoked", "automatically endorsed", and "easily accessible as and when required" (Clark 2010, p. 46), cannot elucidate the causal patterns of selective niche construction and developmental niche construction. The trust and glue criteria, however, do help us understand under what conditions active inference can be said to extend from the individual organism to the cognitive niche. It should be noted, however, that these criteria 
are organism-centred, i.e., they specify the coordination dynamics of an individual organism and its niche.

One way to move beyond this organism-centredness, Constant et al. (2020) propose, is to take work on diachronic extended cognition into consideration (Kirchhoff 2012, 2015). According to this view, cognitive processes extend, in many cases, into a cognitive niche in which organisms jointly engage in patterned, social-cultural practices (Roepstorff, Niewöhner and Beck 2010). It seems to me that Constant et al. (2020) suggest that their extended active inference account of uploading would be entirely consistent with diachronic extended cognition (cf. p. 18). If my analysis of the scope of cognitive uploading is correct, however, it follows that the extended active inference account in its current form does not capture diachronic extended cognition for two reasons. First, uploading refers to selective niche construction and developmental niche construction. The extended active inference account only captures organism-niche coordination dynamics that is synchronic, not diachronic. Second, the current formalisation of extended active inference is organism-centred and therefore does not capture the collective engagement in socio-cultural practices by multiple organisms.

Two steps would be needed, then, to formalise diachronic extended cognition in terms of variational active inference. First, under the assumption that diachronic extended cognition spans across individuals and across developmental time, its proponents would need to show how Variational Active Inference could accommodate the process of developmental niche construction and how this causal pattern relates to organism-niche coordination dynamics. Second, and relatedly, it would need to be shown how the formal apparatus of variational active inference can capture the coordination dynamics of a collective of organisms and their co-constructed niche. Despite the general suggestion by Constant et al. (2020) that a description of the diachronic engagement of collaborating organisms is important for understanding the full scope of extended cognition, as suggested by Kirchhoff (2012), their current version of the extended active inference account remains, on a formal and conceptual level, organism-centred.

This organism-centeredness also seems to characterise Constant's et al. (2020) suggestion to establish a connection between variational active inference and epistemic action. According to Kirsh and Maglio (1994), who first examined and conceptualised this class of actions, epistemic actions are "physical actions that make mental computation easier, faster, or more reliable [...] that an agent performs to change his or her own computational state" (pp. 513-514; for discussions, see Clark 2008; Menary 2010b). Epistemic actions, generally, reduce the "space complexity", "time complexity", and "the probability of error in mental computation" (ibid., p. $514) .^{5}$

\footnotetext{
${ }^{5}$ It is important to appreciate the scientific context of Kirsh and Maglio's (1994) pioneering work in the cognitive sciences. At the time this work was published, classical, internalistic computationalism was still the leading paradigm in the study of cognition. The proposal that epistemic actions, i.e., the embodied manipulation of external resources, directly contribute to problem-solving and other cognitive processes was breaking new ground, paving the way towards philosophical and empirical work on extended cognition, distributed cognition, and cognitive integration.
} 
The reduction of space complexity, time complexity and the probability of errors, Constant et al. (2020) suggest, can be expressed in terms of variational active inference in the following way (p. 17):

Space complexity corresponds to reduced numbers of counterfactual scenarios that one has to model, which naturally entails minimizing the probability of errors (i.e., the more complex the generative model is, the more it is likely to overfit), and by the same token increases performance in terms of time complexity of computation

Note that within Variational Active Inference, space and time complexity express the statistical complexity of the organismic generative model. It is a measure of the difference between Bayesian prior beliefs and the posterior probability of sensory causes (Friston 2010). The generative model will be optimised if its complexity is minimised while maintaining a high level of accuracy (cf. Constant et al. 2020). The variational formalisation, then, gives rise to a new expression of the reduction space complexity, time complexity, and the probability of error as originally defined by Kirsh and Maglio (1994), thereby specifying the contribution of epistemic action to variational organism-niche coordination dynamics.

This assumption can be further substantiated by taking the crucial contribution of the estimation of salience and precision estimation into consideration. Constant et al. (2020) suggest "[...] that neutrally supported estimations of salience (a.k.a., expected surprise) help select actions that can purposefully roll in cognitive operations flowing through bioexternal resources" (p. 17). In other words, salience estimation provides a measure of understanding the selection of specific external resources in the niche for epistemic actions, given current task demands. Furthermore, epistemic actions are modulated, the extended active inference account suggests, by precision estimation. Specifically, “[...] the internal flux of precision (i.e., uncertainty in my beliefs) is resolved by the externally structured flow of epistemic (i.e., salience minimizing) action that serves to improve the long-term fit between by actions and my goals, as well as the cost of computing these long-term goals" (ibid., p. 18). Together, salience estimation and precision estimation, then, structure the ways in which an organism performs epistemic actions by manipulating cognitive resources in the niche. This way of formalising and re-conceptualising epistemic action elucidates, I suggest, the cognitive role of the active manipulation of resources in the niche, thereby specifying an important aspect of organism-niche coordination dynamics.

The upshot is that the extended active inference account formalises and reconceptualises key aspects of the extended cognition thesis. In particular, the parity principle and the functional isomorphism of internal and external states can be cashed out in terms of variational active inference. However, the formal expression of extended active inference is equally consistent with the complementarity principle, which should be preferred, I have argued, on theoretical grounds. In addition, a reliance on the complementarity principle foregrounds the importance of epistemic actions for free energy minimisation under variational active inference: epistemic actions can reduce, as suggested by Constant 
et al. (2020), the space complexity, time complexity, and the probability of errors of the organismic generative model. However, given that the extended active inference account only captures individual-specific organism-niche coordination dynamics, it does not provide, in its current form, a specification of uploading, which is a function of selective niche construction and developmental niche construction. Furthermore, given this focus of the extended active inference account, it does not speak to key aspects of diachronic extended cognition, as this would require a variational description of collective organism-niche coordination dynamics and its relation to developmental niche construction.

\section{Concluding remarks}

In this paper, I have explored the explanatory scope of Variational Active Inference models of the reciprocal causal relationship between organisms and their niche with a focus on the extended active inference account (Constant et al. 2020) and additional considerations on the variational approach to niche construction (Constant et al. 2018). I have proposed, based on Stotz's (2017) analysis, that the notions of selective niche construction, developmental niche construction, and organism-niche coordination dynamics refer to ontologically distinct causal patterns, each of which has a specific scope. I have argued that the extended active inference account, in its current version, only captures organism-niche coordination dynamics and leaves selective niche construction and developmental niche construction, against the goal set by Constant et al. (2020), unexplained. For the time being, I am therefore opting for limiting the explanatory scope of the extended active inference account - and the variational approach to niche construction-given that both interpretations and applications of Variational Active Inference follow the same explanatory strategy and share key commitments. Under the assumption that the extended active inference account can, however, contribute to a better understanding of organism-niche coordination dynamics, I have examined the theoretical implications of integrating Variational Active Inference with the extended cognition thesis. In conclusion, I submit that the extended active inference account can indeed cash out some of the key insights of work on extended cognition, especially the cognitive function of epistemic actions and the complementarity, rather than functional parity, of internal and external states. However, given the focus of the extended active inference account on individual-specific organism-niche coordination dynamics in the here-and-now, more work needs to be done, I suggest, to provide a concise and conceptually coherent account of cognitive uploading and diachronic extended cognition.

Ultimately, this paper offers a standing invitation to the proponents of the extended active inference account and the variational approach to niche construction to take up the challenge to show, on formal and conceptual levels, how Variational Active Inference can explain selective niche construction, developmental niche construction, and collective organism-niche coordination dynamics and how these distinct causal patterns are related under variational active inference. 
Acknowledgement I would like to thank Markus Pantsar for his helpful comments on a previous version of this paper.

\section{Compliance with ethical standards}

Conflict of interest The authors declare that they have no conflict of interest.

Open Access This article is licensed under a Creative Commons Attribution 4.0 International License, which permits use, sharing, adaptation, distribution and reproduction in any medium or format, as long as you give appropriate credit to the original author(s) and the source, provide a link to the Creative Commons licence, and indicate if changes were made. The images or other third party material in this article are included in the article's Creative Commons licence, unless indicated otherwise in a credit line to the material. If material is not included in the article's Creative Commons licence and your intended use is not permitted by statutory regulation or exceeds the permitted use, you will need to obtain permission directly from the copyright holder. To view a copy of this licence, visit http://creativecommons.org/licen ses/by/4.0/.

\section{References}

Bertolotti T, Magnani L (2017) Theoretical considerations on cognitive niche construction. Synthese 194:4757-4779. https://doi.org/10.1007/s11229-016-1165-2

Bruineberg J, Rietveld E, Parr T, van Maanen L, Friston KJ (2018) Free-energy minimization in joint agent-environment systems: a niche construction perspective. J Theor Biol 455:161-178. https:// doi.org/10.1016/j.jtbi.2018.07.002

Clark A (2005) Word, niche, and super-niche. Theoria 54:255-268

Clark A (2006) Language, embodiment, and the cognitive niche. Trends Cogn Sci 10(8):370-374. https:// doi.org/10.1016/j.tics.2006.06.012

Clark A (2008) Supersizing the mind: embodiment, action, and cognitive extension. Oxford University Press, New York

Clark A (2010) Memento's revenge: the extended mind, extended. In: Menary R (ed) The extended mind. MIT Press, Cambridge, Mass, pp 43-66

Clark A (2013) Whatever next? Predictive brains, situated agents, and the future of cognitive science. Behav Brain Sci 36(03):181-204. https://doi.org/10.1017/S0140525X12000477

Clark A (2016) Surfing uncertainty: prediction, action, and the embodied mind. Oxford University Press, Oxford, New York

Clark A, Chalmers D (1998) The extended mind. Analysis 58(1):7-19

Colombo M, Wright C (2018) First principles in the life sciences: the free-energy principle, organicism, and mechanism. Synthese, 1-26. https://doi.org/10.1007/s11229-018-01932-w

Constant A, Clark A, Kirchhoff MD, Friston KJ (2020) Extended active inference: constructing predictive cognition beyond skulls. Mind Lang, 1-22. https://doi.org/10.1111/mila.12330

Constant A, Ramstead MJD, Veissiere SPL, Campbell JO, Friston KJ (2018) A variational approach to niche construction. J R Soc Interface 15(141):1-14. https://doi.org/10.1098/rsif.2017.0685

Craver CF (2015) Levels. In: Metzinger TK, Windt JM (eds) Open MIND. Frankfurt am Main: MIND Group, pp. 1-26. https://doi.org/10.15502/9783958570498

DiFrisco J (2017) Time scales and levels of organization. Erkenntnis 82(4):795-818. https://doi. org/10.1007/s10670-016-9844-4

Eronen MI (2015) Levels of organization: a deflationary account. Biol Philos 30(1):39-58. https://doi. org/10.1007/s10539-014-9461-z

Fabry RE (2018) Betwixt and between: the enculturated predictive processing approach to cognition. Synthese 195(6):2483-2518. https://doi.org/10.1007/s11229-017-1334-y

Fabry RE (2018) Enculturation and narrative practices. Phenomeno Cogn Sci 17(5):911-937. https://doi. org/10.1007/s11097-018-9567-X 
Fabry RE (2020) The cerebral, extra-cerebral bodily, and socio-cultural dimensions of enculturated arithmetical cognition. Synthese 197(9):3685-3720. https://doi.org/10.1007/s11229-019-02238-1

Flynn EG, Laland KN, Kendal RL, Kendal JR (2013) Developmental niche construction. Dev Sci 16(2):296-313. https://doi.org/10.1111/desc.12030

Friston K (2010) The free-energy principle: a unified brain theory? Nat Rev Neurosci 11(2):127-138. https://doi.org/10.1038/nrn2787

Friston K (2013) Active inference and free energy. Behav Brain Sci 36(03):212-213. https://doi. org/10.1017/S0140525X12002142

Friston K (2013) Life as we know it. J R Soc Interface 10(86):1-12

Friston K, Rigoli F, Ognibene D, Mathys C, Fitzgerald T, Pezzulo G (2015) Active inference and epistemic value. Cogn Neurosci 6(4):187-214

Friston K, Sengupta B, Auletta G (2014) Cognitive dynamics: from attractors to active inference. Proc IEEE 102(4):427-445. https://doi.org/10.1109/JPROC.2014.2306251

Griffiths PE, Hochman A (2015) Developmental systems theory. In: Encyclopedia of life sciences. John Wiley \& Sons, New Jersey. pp. 1-7. https://doi.org/10.1002/9780470015902.a0003452.pub2

Griffiths P, Stotz K (2018) Developmental systems theory as a process theory. In: Nicholson DJ, Dupré J (eds) Everything flows: towards a processual philosophy of biology. Oxford University Press, Oxford, pp 225-245

Hesp C, Ramstead M, Constant A, Badcock P, Kirchhoff M, Friston K (2019) A multi-scale view of the emergent complexity of life: a free-energy proposal. In: Georgiev GY, Smart JM, Flores Martinez CL, Price ME (eds) Evolution, development and complexity. Cham, Springer International Publishing, pp 195-227

Heyes C (2012) Grist and mills: on the cultural origins of cultural learning. Philo Trans Royal Soc B: Biol Sci 367(1599):2181-2191. https://doi.org/10.1098/rstb.2012.0120

Heyes C (2018) Cognitive gadgets: the cultural evolution of thinking. Harvard University Press, Cambridge, Mass.

Hohwy J (2013) The predictive mind. Oxford University Press, Oxford

Hohwy J (2015) The neural organ explains the mind. In: Metzinger T, Windt JM (eds) Open MIND. Frankfurt am Main,MIND Group, pp 1-22. https://doi.org/10.15502/9783958570016

Hohwy J (2020) Self-supervision, normativity and the free energy principle. Synthese, 1-25. https://doi. org/10.1007/s11229-020-02622-2

Jones M (2020) Numerals and neural reuse. Synthese 197(9):3657-3681. https://doi.org/10.1007/s1122 9-018-01922-y

Kirchhoff MD (2012) Extended cognition and fixed properties: steps to a third-wave version of extended cognition. Phenomenol Cogn Sci 11(2):287-308. https://doi.org/10.1007/s11097-011-9237-8

Kirchhoff MD (2015) Extended cognition \& the causal-constitutive fallacy: in search for a diachronic and dynamical conception of constitution. Res 90(2):320-360. https://doi.org/10.1111/phpr.12039

Kirsh D, Maglio P (1994) On distinguishing epistemic from pragmatic action. Cogn Sci 18(4):513-549. https://doi.org/10.1207/s15516709cog1804_1

Laland KN, O’Brien MJ (2011) Cultural niche construction: an introduction. Biol Theory 6(3):191-202

Laland KN, Odling-Smee J, Feldman MW (2000) Niche construction, biological evolution, and cultural change. Behav Brain Sci 23(1):131-146

Menary R (2007) Writing as thinking. Lang Sci 29(5):621-632. https://doi.org/10.1016/j.langs ci.2007.01.005

Menary R (2010) Cognitive integration and the extended mind. In: Menary R (ed) The extended mind. MIT Press, Cambridge, Mass, pp 227-243

Menary R (2010) Dimensions of mind. Phenomenol Cogn Sci 9(4):561-578. https://doi.org/10.1007/ s11097-010-9186-7

Menary R (2013) Cognitive integration, enculturated cognition and the socially extended mind. Cogn Syst Res 25-26:26-34. https://doi.org/10.1016/j.cogsys.2013.05.002

Menary R (2014) Neural plasticity, neuronal recycling and niche construction. Mind Lang 29(3):286303. https://doi.org/10.1111/mila.12051

Menary R (2015) Mathematical cognition: a case of enculturation. In: Metzinger T, Windt JM (eds) Open MIND, Frankfurt am Main: MIND Group, pp 1-20. https://doi.org/10.15502/9783958570818

Menary R, Gillett AJ (2016) Embodying culture: integrated cognitive systems and cultural evolution. In: Kiverstein J (ed) The Routledge handbook of philosophy of the social mind. Routledge, London, New York, pp 88-103 
Odling-Smee J, Laland KN (2011) Ecological inheritance and cultural inheritance: what are they and how do they differ? Biolo Theory 6(3):220-230

Oppenheim P, Putnam H (1958) Unity of science as a working hypothesis. In: Feigl H, Scriven M, Maxwell G (eds) Minnesota studies in the philosophy of science. University of Minnesota Press, Minneapolis, pp 3-36

Pantsar M (2019) The enculturated move from proto-arithmetic to arithmetic. Front Psychol 10:1-15. https://doi.org/10.3389/fpsyg.2019.01454

Potochnik A (2017) Idealization and the aims of science. The University of Chicago Press, Chicago

Potochnik A, McGill B (2012) The limitations of hierarchical organization. Philo Sci 79(1):120-140. https://doi.org/10.1086/663237

Potochnik A, Sanches de Oliveira G (2020) Patterns in cognitive phenomena and pluralism of explanatory styles. Topics Cogn Sci 12:1306-1320. https://doi.org/10.1111/tops.12481

Ramstead MJD, Constant A, Badcock PB, Friston KJ (2019) Variational ecology and the physics of sentient systems. Phys Life Rev 31:188-205. https://doi.org/10.1016/j.plrev.2018.12.002

Ramstead MJD, Veissière SPL, Kirmayer LJ (2016) Cultural affordances: scaffolding local worlds through shared intentionality and regimes of attention. Front Psychol 7:1-21

Ramstead MJD, Badcock PB, Friston KJ (2018) Answering Schrödinger's question: a free-energy formulation. Phys Life Rev 24:1-16

Risko EF, Gilbert SJ (2016) Cognitive offloading. Trends Cogn Sci 20(9):676-688

Roepstorff A, Niewöhner J, Beck S (2010) Enculturing brains through patterned practices. Neural Netw 23(8-9):1051-1059. https://doi.org/10.1016/j.neunet.2010.08.002

Sterelny K (2003) Thought in a hostile world: the evolution of human cognition. Blackwell, Malden, Mass.

Sterelny K (2010) Minds: extended or scaffolded? Phenomenol Cogn Sci 9(4):465-481. https://doi. org/10.1007/s11097-010-9174-y

Sterelny K (2012) The evolved apprentice: how evolution made humans unique. MIT Press, Cambridge, Mass.

Stotz K (2010) Human nature and cognitive-developmental niche construction. Phenomenol Cogn Sci 9(4):483-501. https://doi.org/10.1007/s11097-010-9178-7

Stotz K (2017) Why developmental niche construction is not selective niche construction: and why it matters. Interface Focus 7(5):1-10. https://doi.org/10.1098/rsfs.2016.0157

Sutton J (2010) Exograms and interdisciplinarity: history, the extended mind, and the civilizing process. In: Menary R (ed) The extended mind. MIT Press, Cambridge, Mass, pp 189-225

Veissière SPL, Constant A, Ramstead MJD, Friston KJ, Kirmayer LJ (2020) Thinking through other minds: a variational approach to cognition and culture. Behav Brain Sci 43:1-22. https://doi. org/10.1017/S0140525X19001213

West MJ, King AP (1987) Settling nature and nurture into an ontogenetic niche. Dev Psychobiol 20(5):549-562

Publisher's Note Springer Nature remains neutral with regard to jurisdictional claims in published maps and institutional affiliations. 